\title{
SOME INEQUALITIES THAT ARISE IN MEASURE THEORY
}

\author{
GAVIN BROWN
}

(Received 4 May 1987)

Communicated by J. F. Price \begin{abstract}
Dedicated to Robert Edwards in recognition of
25 years' distinguished contribution to mathematics in Australia, on the occasion of his retirement
\end{abstract}

\begin{abstract}
The Lebesgue measure, $\lambda(E+F)$, of the algebraic sum of two Borel sets, $E, F$ of the classical "middle-thirds" Cantor set on the circle can be estimated by evaluating the Cantor measure, $\mu$, of the summands. For example $\log \lambda(E+F)$ exceeds a fixed scalar multiple of $\log \mu(E)+$ $\log \mu(F)$. Several numerical inequalities which are required to prove this and related results look tantalizingly simple and basic. Here we isolate them from the measure theory and present a common format and proof.
\end{abstract}

1980 Mathematics subject classification (Amer. Math. Soc.) (1985 Revision): primary 26 D 20; secondary 43 A 05,43 A 10.

\section{Introduction}

It is reasonably common for a measure-theoretic estimate to reduce to an inequality involving only real numbers. That inequality is, in turn, usually some variant of one of the classical inequalities or else so specialized as to be clearly an ad hoc exercise. Recent investigations of singular measures have led to simple inequalities such as

$$
1+x+x^{2} \geq\left(1+x^{s}\right)^{2 / s}, \quad x>0, s=\log _{3} 4,
$$

(C) 1988 Australian Mathematical Society $0263-6115 / 88 \$ A 2.00+0.00$ 
which look fundamental but do not appear to belong to any of the standard classes of known inequalities (see [1], [9], [10]). While their proofs are not exceptionally difficult, such inequalities are sufficiently "tight" that it appears worthwhile to subject them to independent study.

The hope is, of course, that freed from the measure theory context, patterns and proofs will stand out more boldly. There have already been gains. Each of the propositions of the next section is a new result (which can be fed back into the measure theory). Moreover our proofs offer a shorter path to known results. It will be clear to the reader, however, that there remains considerable scope for extension.

Part of this work was developed while the author visited the University of Cambridge and he thanks S.E.R.C and D.P.M.M.S. in general and Tom Korner in particular for kind hospitality.

Before specializing the discussion to specific inequalities and their proofs, we should discuss some background. As it happens, the Cantor sum set problem described in the preceding abstract has an amusing history.

Motivated by consideration of Raikov systems for convolution measure algebras (for general background see Graham and McGehee [6]) W. Moran and the author formally proposed the sum set problem in the book of the 1974 LMS Durham Research Symposium on Functional Analysis and Stochastic Processes. We conjectured that the sum of two Borel sets of positive Cantor measure has positive Lebesgue measure and stated that the proof could be reduced to a counting problem concerning mid-points of joins of vertices of hypercubes. We had been unable to reduce either problem to a convenient analytic inequality.

The basic sum set problem was soon solved by M. Talagrand [12] who attributed it to R. Haydon. The counting problem was taken up by R. R. Hall [8] and then D. R. Woodall [13], Woodall formulated and proved the fundamental inquality: for $0 \leq x, y \leq 1, \alpha=\log _{4} 3$,

$$
x^{\alpha} y^{\alpha}+\max \left(x^{\alpha}(1-y)^{\alpha}, y^{\alpha}(1-x)^{\alpha}\right)+(1-x)^{\alpha}(1-y)^{\alpha} \geq 1 .
$$

Moran and I then showed, in [3], how Woodall's inequality gives

$$
\lambda(E+F) \geq \mu(E)^{\alpha} \mu(F)^{\alpha}
$$

and we discussed refinements of the original Banach algebra problem.

Meanwhile D. Hajela and P. Seymour, [7], unaware of the work of Hall and Woodall, but knowing Talagrand [12], gave an independent derivation of both (2) and (3). They also solved the hypercube problem, but attributed it to Erdös, and referred to our original Raikov system problem, but attributed it to Moran alone.

Hajela and Seymour mention the obvious $n$-summand generalization of (3) but are unable to verify it. That result proved surprisingly tricky (a more general 
but false statement appears on page 182 of [6]) and was finally established in Brown, Keane, Moran and Pearce [2] after some special cases had been dealt with by R. Bott, a student of Moran.

Meanwhile D. M. Oberlin had been developing sum set results of the type

$$
\delta \lambda(E)^{\beta} \leq \lambda(E+K)
$$

where $K$ is some fixed measurable null set and $\delta>0, \beta \in(0,1)$ depend on $K$. In Oberlin [11], $\lambda$ is Haar measure on a locally compact abelian group and the motivation comes from the study of certain $k$-dimensional surfaces $K$ in $\mathbf{R}^{n}=G$, $1 \leq k<n$. Oberlin [11] considers (4) when $K$ is a Cantor set. Then he relates his methods to those of Brown and Moran [3]. In particular he establishes the inequality

$$
\max \left(a_{0} x, a_{1}(1-x)\right)+\max \left(a_{1} x, a_{2}(1-x)\right)+\max \left(a_{2} x, a_{0}(1-x)\right) \geq 1,
$$

for $0 \leq x \leq 1, a_{i} \geq 0, a_{0}^{1 / \beta}+a_{1}^{1 / \beta}+a_{2}^{1 / \beta}=1, \beta=1-\log _{3} 2$, and uses it to prove

$$
\mu(E) \lambda(F)^{\beta} \leq \lambda(E+F),
$$

for $\mu$ Cantor measure and $\lambda$ Haar measure on the circle.

Inequality (5) with its side conditions may seem to call in question our remark about "inequalities which look tantalizingly simple and basic". Indeed we shall work with simpler versions of (2), (5) which retain the form of (3), (6) more clearly. In this sense the simplified version of $(2)$ is (1) and the simplified version of (5) appears in the statement of Proposition 2, below.

Our simplification is not without cost. There is extra work involved in deriving measure theoretic results from the simplified canonical forms of the inequalities. Going from (1) to (2) is quite easy but the corresponding step for a more complex inequality can require effort. (The arguments up to and including Lemma 1 of [2] give a non-trivial example.)

We leave the detailed discussion of the passage from canonical inequalities to measure-theoretic results for another occasion [5]. Suffice it to say that such a passage is possible in several cases discussed here. Thus Proposition 1 extends (2), Proposition 2 extends (6) and, for example, Proposition 3(i) yields the following result.

Suppose that the probability measure $\nu$ is defined on the circle (identified with $[0,1)$ ) as the distribution of a number whose base 4 expansion is random except that only the digits 0,1 , are used. Then

$$
\nu(E)^{\alpha} \lambda(F)^{\beta} \leq \lambda(E+F)
$$

whenever $0 \leq \alpha \leq 1,0 \leq \beta \leq 1, \frac{1}{2} \alpha+\beta \leq 1$. (In this context note that the Cantor measure $\mu$ appearing in (2), (6) can be realized as the distribution of a number whose base 3 expansion is random except that only the digits 0,2 are used.) 
The remaining cases of Proposition 3 do not transfer so readily.

\section{The inequalities}

The proofs that follow are exercises in elementary calculus with the twist that we must handle sums of fractional powers rather than polynomials. We have found significant shortcuts for the proofs of Propositions 2, 3 but the proof of Proposition 1 is still rather long.

PROPOSITION 1. Suppose that $s, t \geq 1$ and that $s^{-1}+t^{-1}=\log 3 / \log 2$. Then,

$$
1+x+x^{2} \geq\left(1+x^{s}\right)^{1 / s}\left(1+x^{t}\right)^{1 / t},
$$

for all $0 \leq x \leq 1$, if and only if

$$
3(s+t) \leq 8 .
$$

ProOF. Note that the standing assumption on $s, t$ corresponds to equality at $x=1$. Let's check necessity of the other condition on $s, t$. In fact consider $x=1-y$, for small positive $y$, and note that

$$
\begin{aligned}
\left(1+x^{s}\right)^{1 / s} & =2^{1 / s}\left(1-(s / 2) y+(s(s-1) / 4) y^{2}+O\left(y^{3}\right)\right)^{1 / s} \\
& =2^{1 / s}\left(1-(y / 2)+((s-1) / 8) y^{2}+O\left(y^{3}\right)\right) .
\end{aligned}
$$

It follows that

$$
\left(1+x^{s}\right)^{1 / s}\left(1+x^{t}\right)^{1 / t}=2^{s^{-1}+t^{-1}}\left(1-y+((s+t) / 8) y^{2}+O\left(y^{3}\right)\right)
$$

and this is to be compared with

$$
1+x+x^{2}=3-3 y+y^{2} .
$$

The required condition is derived by considering the coefficients of $y^{2}$.

Now we would like to reduce to consideration of specific $s, t$. In fact if $3(s+t)=$ $8, s^{-1}+t^{-1}=\log 3 / \log 2$, and $s<t$, then $s=1.0246 \ldots$ and $t=1.6420 \ldots$ are uniquely determined. Moreover, for any $s_{1}, t_{1}$ such that $s_{1}^{-1}+t_{1}^{-1}=\log 3 / \log 2$ and $3\left(s_{1}+t_{1}\right) \leq 8$ we must have $s \leq s_{1} \leq t$. From this it is possible to deduce that

$$
\left(1+x^{s_{1}}\right)^{1 / s_{1}}\left(1+x^{t_{1}}\right)^{1 / t_{1}} \leq\left(1+x^{s}\right)^{1 / s}\left(1+x^{t}\right)^{1 / t} .
$$

The last step uses a simple observation by Larry Shepp and the present author ([4], Lemma 4) to the effect that, for a fixed function $f, \log \|f\|_{s}+\log \|f\|_{t(s)}$ (where $s / t(s)$ is affine) has no local maxima as a function of $s$. 
The last paragraph has shown that we need only prove the inequality for the special values of $s, t$ mentioned earlier (that is, $s=1.024 \ldots, t=1.642 \ldots$ ) Accordingly we fix $s, t$ and proceed. We write

$$
F(x)=\left(1+x+x^{2}\right) /\left(1+x^{s}\right)^{1 / s}\left(1+x^{t}\right)^{1 / t} .
$$

Because $F(0)=F(1)=1$ and $F$ is greater than 1 for small positive $x$ it will suffice to prove that $F^{\prime}$ has precisely one zero for $0<x<1$. Considering the derivative of $\log F$, we see that $F^{\prime}$ is a positive multiple of the function $G$ defined by

$$
\begin{aligned}
G(x)= & (1+2 x)\left(1+x^{s}\right)\left(1+x^{t}\right) \\
& -x^{s-1}\left(1+x+x^{2}\right)\left(1+x^{t}\right)-x^{t-1}\left(1+x+x^{2}\right)\left(1+x^{s}\right) \\
= & 1+2 x-x^{s-1}-x^{t-1}+x^{s+1}+x^{t+1}-2 x^{s+t-1}-x^{s+t} .
\end{aligned}
$$

Observe that $G(0)=1, G(1)=0$. Therefore it will, in turn, suffice to prove that $G^{\prime}$ has precisely one zero for $0<x<1$. Unfortunately successive differentiation of $G$ does not appear to clarify this point, so we are led to consider rather $x^{1+s-t}\left[-x^{2-s} G^{\prime}(x)\right]^{\prime}$. Tackling the problem in stages, let's write

$$
\begin{aligned}
H(x)= & -x^{2-s} G^{\prime}(x) \\
= & (s-1)+(t-1) x^{t-s}-2 x^{2-s}+2(s+t-1) x^{t} \\
& -(s+1) x^{2}-(t+1) x^{t-s+2}+(s+t) x^{t+1} .
\end{aligned}
$$

Note that $H(0)=s-1>0, H^{\prime}\left(0_{+}\right)>0$, while $H(1)=3(s+t)-8=0$. Thus to prove that $H$ (hence $G^{\prime}$ ) has precisely one zero for $0<x<1$, it will suffice to establish that $H^{\prime}$ has precisely two zeros for $0<x<1$. Let us consider rather $K$ defined by

$$
\begin{aligned}
K(x)= & x^{1+s-t} H^{\prime}(x) \\
= & (t-1)(t-s)-2(2-s) x^{2-t}+2 t(s+t-1) x^{s} \\
& -(t+1)(t-s+2) x^{2}+(s+t)(t+1) x^{s+1}-2(s+1) x^{2+s-t} .
\end{aligned}
$$

Observe that $K(0)=(t-1)(t-s)>0$, while $K(1)=0$, as we now check:

$$
\begin{aligned}
(t-1)(t-s) & -2(2-s)+2 t(s+t-1) \\
& -(t+1)(t-s+2)+(s+t)(t+1)-2(s+1) \\
= & 3 t^{2}+3 s t-5 t+3 s-8 \\
= & 3 t^{2}+3 s t-8 t+3 t+3 s-8 \\
= & (t+1)(3 t+3 s-8)=0 .
\end{aligned}
$$

We have

$$
\begin{aligned}
K^{\prime}(x)= & -2(2-s)(2-t) x^{1-t}+2 s t(s+t-1) x^{s-1} \\
& -2(s+1)(2+s-t) x^{1+s-t} \\
& -2(t+1)(t-s+2) x+(s+t)(t+1)(s+1) x^{s} .
\end{aligned}
$$




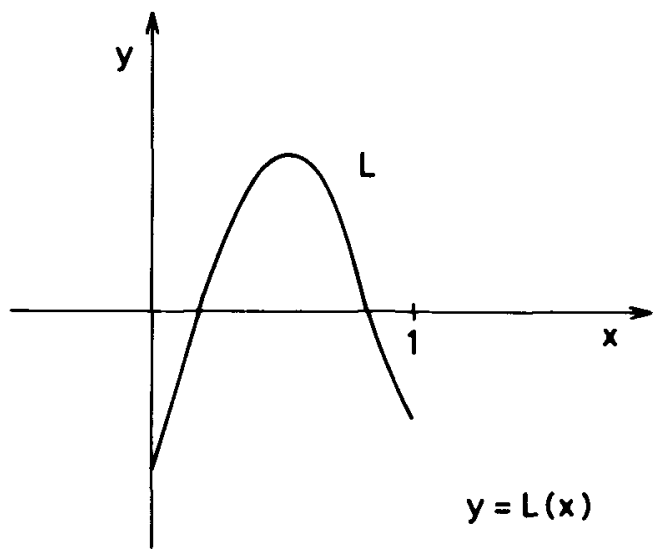

Figure I

and introduce $L$ defined by

$$
L(x)=x^{t-1} K^{\prime}(x)
$$

It will turn out that $L^{\prime \prime}$ is negative for $0<x \leq 1$. In fact

$$
\begin{aligned}
L^{\prime}(x)= & 2 s t(s+t-1)(s+t-2) x^{s+t-3}-2(s+1)(2+s-t) s x^{s-1} \\
& -2(t+1)(t-s+2) t x^{t-1}+(s+t)(t+1)(s+1)(s+t-1) x^{s+t-2}, \\
L^{\prime \prime}(x)= & 2 s t(s+t-1)(s+t-2)(s+t-3) x^{s+t-4} \\
& -2(s+1)(2+s-t) s(s-1) x^{s-2} \\
& -2(t+1)(t-s+2) t(t-1) x^{t-2} \\
& +(s+t)(t+1)(s+1)(s+t-1)(s+t-2) x^{s+t-3} .
\end{aligned}
$$

It is clear that the terms in the expression for $L^{\prime \prime}$ are arranged in ascending powers of $x$ and that the first three coefficients are negative. Accordingly to prove that $L^{\prime \prime}$ is negative throughout the interval ]0,1] it is enough to check that $L^{\prime \prime}(1)$ is negative. Using the estimates $1.024<s<1.025,1.642<t<1.643$, $3(s+t)=8$, we see, in fact, that $L^{\prime \prime}(1)<-0.102$.

Now we can give the shape of all the curves involved. Since $H(0)>0$, $H(1)=0$ we know that $H^{\prime}(x)<0$ for some $0<x<1$ and hence that $K$ is somewhere negative. Since $K(1)=0$ this shows that $K^{\prime}$ is strictly positive for some $0<x<1$ and hence so is $L$. However $L(0)=-2(2-s)(2-t)<0$ and direct calculation shows that $L(1)<0$ (The sum of the negative terms has absolute value in excess of 20 and the sum of the positive terms is strictly less than 20.) Some rough sketches may be helpful at this point.

Figure I shows the general shape of $L$. In fact $L^{\prime}$ has precisely one zero and $L$ has precisely two, for $0 \leq x \leq 1$. 


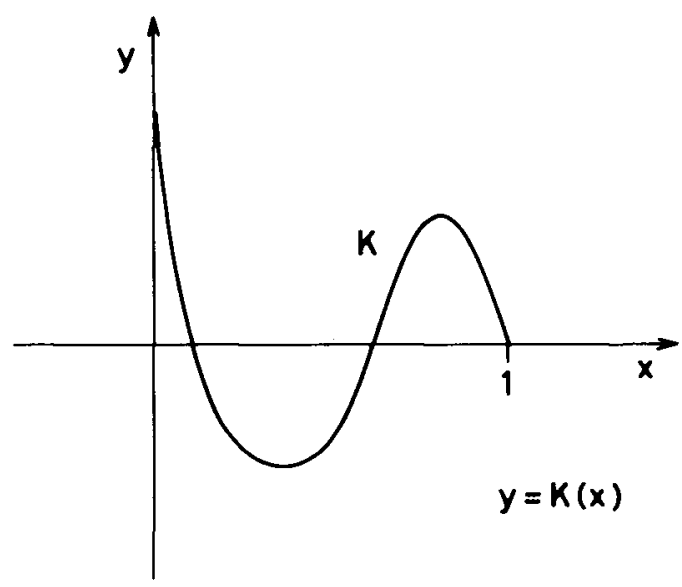

Figure II

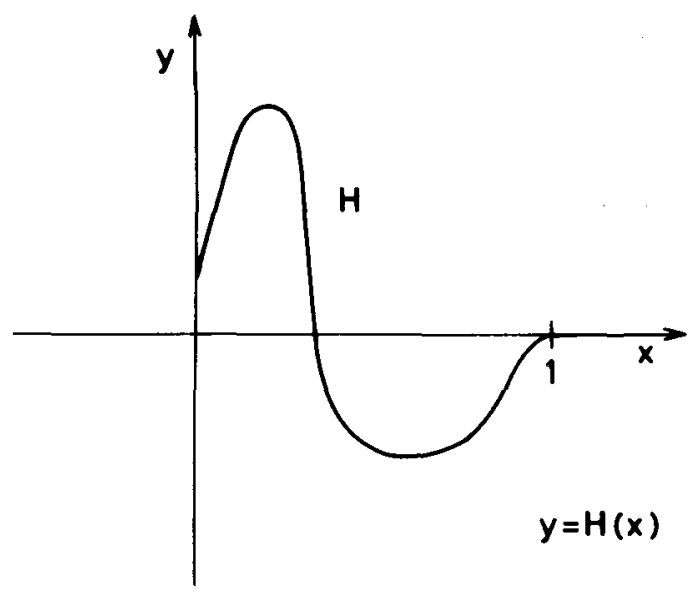

Figure III

Armed with this information concerning $L$, and hence $K^{\prime}$, we are able to determine the general shape of the graph of $K$. See Figure II. The function $K$ has exactly two critical points-a local minimum followed by a local maximum. Since $K(1)=0, K^{\prime}(1)>0$ we see that the local maximum occurs for positive $K$. On the other hand we previously noted that $K$ is somewhere negative--so the local minimum is, in fact, the minimum for $0 \leq x \leq 1$ and occurs below the $x$-axis.

Now we turn to $H$ and Figure III. $H(0)$ is positive and $H$ increases further to a maximum before decreasing to a local minimum then increasing once more 
to $0=H(1)$, where the graph is tangent to the horizontal. It is clear therefore that $H$ has precisely one zero for $0<x<1$.

It follows that $G^{\prime}$, and in turn $G$, has precisely one zero for $0<x<1$ and this completes the proof.

Proposition 2. Suppose that $s, t \geq 1$ and that $\left(1-t^{-1}\right) \log 3=s^{-1} \log 2$. Then

$$
1+x+x^{2} \geq\left(1+x^{s}\right)^{1 / s}\left(1+x^{t}+x^{2 t}\right)^{1 / t},
$$

for all $0 \leq x \leq 1$.

PROOF. Once more the standing assumption on $s, t$ corresponds to equality for $x=1$. Moreover the relationship

$$
t(s)^{-1}+(\log 2 / \log 3) s^{-1}=1
$$

is such that $s / t(s)$ is affine in $s$. Thus Lemma 4 of [4] applies and we deduce that the product

$$
\left(1+x^{s}\right)^{1 / s}\left(1+x^{t}+x^{2 t}\right)^{1 / t}
$$

is majorized by the maximum of the corresponding products for the special cases $s=1, t=(1-(\log 2 / \log 3))^{-1} ; s=\infty, t=1$. The case $s=\infty, t=1$ reduces to equality because

$$
\left(1+x^{s}\right)^{1 / s} \rightarrow 1
$$

(recall that $0 \leq x \leq 1$ ).

Thus we have reduced the problem to the special case $s=1, t=\log 3 / \log (3 / 2)$ $=2.7095 \ldots$ As before we consider a suitable function $F$; here

$$
F(x)=\left(1+x+x^{2}\right) /(1+x)\left(1+x^{t}+x^{2 t}\right)^{1 / t} .
$$

We note that $F(0)=F(1)=1$ and that $F$ is greater than 1 for small positive $x$. We will show that $F^{\prime}$ has precisely one zero for $0<x<1$. Considering the derivative of $\log F$, we see that $F^{\prime}$ is a positive multiple of $G$ defined by

$$
\begin{aligned}
G(x)= & (1+2 x)(1+x)\left(1+x^{t}+x^{2 t}\right)-\left(1+x+x^{2}\right)\left(1+x^{t}+x^{2 t}\right) \\
& -\left(x^{t-1}+2 x^{2 t-1}\right)(1+x)\left(1+x+x^{2}\right) .
\end{aligned}
$$

It will suffice to show that the function $H$, defined by

$$
H(x)=x^{-1} G(x)=2-x^{t-2}+x-2 x^{t-1}-2 x^{2 t-2}-4 x^{2 t-1}-2 x^{2 t}-x^{2 t+1},
$$

has precisely one zero for $0<x<1$. It is convenient to choose $C=(3-t) /(t-1)$ and write

$$
H(x)=K(x)-L(x)
$$

where

$$
K(x)=2-x^{t-2}+x-C x^{t-1}
$$


Because $0<C<1$, we see that $L$ is strictly increasing throughout $0<x<$ 1. Thus to check that $H$ has only one zero it will suffice to check that $K$ is decreasing. In fact

$$
K^{\prime}=-(t-2) x^{t-3}+1-C(t-1) x^{t-2},
$$

so that $K^{\prime}\left(0_{+}\right)<0$ and $K^{\prime}(1)=-(t-2)+1+(t-3)=0$; while

$$
\begin{aligned}
K^{\prime \prime} & =-(t-2)(t-3) x^{t-4}-C(t-1)(t-2) x^{t-2} \\
& \geq-(t-2)[(t-3)+(3-t)] x^{t-2}=0 .
\end{aligned}
$$

This completes the proof.

REMARK. For the result proved in the last part of the previous proof we could have appealed to the inequality proved in the last theorem of [11] (that is, inequality (5) of the previous section). In fact the argument here leads to a quicker proof of (5) and emphasizes the common theme of the various inequalities being discussed.

We have considered inequalities with $1+x+x^{2}$ as the majorant. These corresponded to applications involving the triadic Cantor measure. For applications related to base four expansions we should use the majorant $1+x+x^{2}+x^{3}=$ $(1+x)\left(1+x^{2}\right)$.

PROPOSITION 3. In each case the stated expression is majorised by $1+x+$ $x^{2}+x^{3}$ for all $0 \leq x \leq 1$ :

(i) $\left(1+x^{s}\right)^{1 / s}\left(1+x^{t}+x^{2 t}+x^{3 t}\right)^{1 / t}$, whenever $\frac{1}{2} s^{-1}+t^{-1}=1 ; s, t \geq 1$;

(ii) $\left(1+x^{s}+x^{2 s}\right)^{1 / s}\left(1+x^{t}+x^{2 t}\right)^{1 / t}$, whenever $\left(\log _{4} 3\right)\left(s^{-1}+t^{-1}\right)=1 ; s, t \geq 1$;

(iii) $\left(1+x^{s}+x^{2 s}\right)^{1 / s}\left(1+x^{t}+x^{2 t}+x^{3 t}\right)^{1 / t}$, whenever $\left(\log _{4} 3\right) s^{-1}+t^{-1}=$ $1 ; s, t \geq 1$

REMARK. Note that in each case we allow all values of $s, t$ so that the expression equals 4 (the value of $1+x+x^{2}+x^{3}$ ) at $x=1$, so the results cannot be improved.

PROOF. In each case it will be possible to apply Lemma 4 of [4] so we need verify the inquality only for the end-point values of $s, t$.

For (i), we consider $s=\infty, t=1$ (which corresponds to a valid equality) and $s=1, t=2$. The latter corresponds to the statement

$$
(1+x)\left(1+x^{2}\right)^{1 / 2}\left(1+x^{4}\right)^{1 / 2} \leq(1+x)\left(1+x^{2}\right),
$$

which is clearly true for $0 \leq x \leq 1$.

Cases (ii), (iii) require more effort. For (ii) we must consider $s=1, t=$ $(\log 4-\log 3) / \log 3=3.8188 \ldots$ (and the corresponding case with $s, t$ interchanged). Proceeding as on earlier occasions we differentiate

$$
\left(1+x+x^{2}+x^{3}\right)\left(1+x+x^{2}\right)^{-1}\left(1+x^{t}+x^{2 t}\right)^{-1 / t}
$$


to see that we should consider zeros of $G(x)$ defined by

$$
\begin{aligned}
G(x)= & \left(1+2 x+3 x^{2}\right)\left(1+x+x^{2}\right)\left(1+x^{t}+x^{2 t}\right) \\
& -\left(1+x+x^{2}+x^{3}\right)(1+2 x)\left(1+x^{t}+x^{2 t}\right) \\
& -\left(1+x+x^{2}+x^{3}\right)\left(1+x+x^{2}\right)\left(x^{t-1}+2 x^{2 t-1}\right) .
\end{aligned}
$$

Then

$$
\begin{aligned}
x^{-2} G(x)= & 3-x^{t-3}+2 x-2 x^{t-2}+x^{2}-3 x^{t-1}-2 x^{2 t-3} \\
& -4 x^{2 t-2}-6 x^{2 t-1}-3 x^{2 t}-2 x^{2 t+1}-x^{2 t+2},
\end{aligned}
$$

and it will suffice to show that this function has precisely one zero for $0 \leq x \leq 1$. Consider $H, K$ defined by

$$
\begin{aligned}
& H(x)=x^{-2} G(x)+x^{t-3}-1, \\
& K(x)=x^{t-3}-1 .
\end{aligned}
$$

To obtain a zero of $G$ we must have $H=K . K$ is negative increasing for $0 \leq x<1$, so it will suffice to show that $H$ is decreasing wherever it is negative. Since $H(0)=2, H^{\prime}(0)=2$, this will indeed be the case provided $H^{\prime}$ has precisely one zero for $0 \leq x \leq 1$. (For future reference note that this reduction has used only the information that $t>3$.) We write

$$
H^{\prime}(x)=2\left(1-(t-2) x^{t-3}+x\right)-L(x),
$$

and note that $L$ is increasing. It will suffice to show that $M$, defined by

$$
M(x)=1-(t-2) x^{t-3}+x,
$$

is decreasing for $0 \leq x \leq 1$. Now we note that

$$
M^{\prime \prime}(x)=-(t-2)(t-3)(t-4) x^{t-5},
$$

so that $M^{\prime}$ increases $(t$ lies between 3 and 4) but

$$
M^{\prime}(1)=1-(t-2)(t-3)<0,
$$

so $M$ is indeed decreasing. This completes the proof of (ii).

We turn to (iii). One extreme case, $s=\infty, t=1$, is easy and we must focus on the case $s=1, t=\left(1-\log _{4} 3\right)^{-1}=4.8188 \ldots$ (Of course this value of $t$ is precisely one more than the value of $t$ considered in case (ii).) We set about differentiating

$$
\left(1+x+x^{2}+x^{3}\right) /\left(1+x+x^{2}\right)\left(1+x^{t}+x^{2 t}+x^{3 t}\right)^{1 / t}
$$

and consider zeros of $G$ given by

$$
\begin{aligned}
G(x)= & \left(1+2 x+3 x^{2}\right)\left(1+x+x^{2}\right)\left(1+x^{t}+x^{2 t}+x^{3 t}\right) \\
& -(1+2 x)\left(1+x+x^{2}+x^{3}\right)\left(1+x^{t}+x^{2 t}+x^{3 t}\right) \\
& -\left(1+x+x^{2}\right)\left(x^{t-1}+2 x^{2 t-1}+3 x^{3 t-1}\right)\left(1+x+x^{2}+x^{3}\right) .
\end{aligned}
$$


We have

$$
\begin{aligned}
x^{-2} G(x)= & +2 x-x^{t-3}+x^{2}-2 x^{t-2}-3 x^{t-1}-2 x^{2 t-3}-4 x^{2 t-2} \\
& -6 x^{2 t-1}-3 x^{2 t}-2 x^{2 t+1}-x^{2 t+2}-3 x^{3 t-3}-6 x^{3 t-2} \\
& -9 x^{3 t-1}-6 x^{3 t}-4 x^{3 t+1}-2 x^{3 t+2}
\end{aligned}
$$

It is slightly misleading that the first several leading terms are formally the same as those for case (ii). The fact that $t$ is different changes the nature of the function - a point we have emphasised by arranging the first few powers in ascending order. Nevertheless we can repeat part of the earlier argument. Using only the fact that $t>3$, we reduce the problem to consideration of the derivative of $H$, where

$$
H(x)=x^{-2} G(x)-1+x^{t-3} .
$$

In our present situation, $t$ is greater than 4 so we are able to note further that

$$
H^{\prime}(x)=2+2 x-\sum a_{i} x^{b_{i}}
$$

where $a_{1}>0$ and $b_{i}>1$. Thus $H^{(3)}(x)<0$, for $0<x \leq 1$, and $H^{\prime}(0)=$ $H^{\prime \prime}(0)=2$. it follows that, in the stated domain, $H^{\prime \prime}$ has at most one zero (of course it does have one) and the proof is complete.

The author thanks David Wilson for checking and in one case correcting the numerical assertions made in the proofs in this section.

\section{References}

[1] E. F. Beckenbach and R. Bellman, Inequalities (Springer-Verlag, Berlin, 1971).

[2] G. Brown, M. S. Keane, W. Moran and C. E. M. Pearce, 'An inequality, with applications to Cantor measures and normal numbers', preprint.

[3] G. Brown and W. Moran, 'Raikov systems and radicals in convolution measure algebras', J. London Math. Soc. (2), 28 (1983), 531-542.

[4] G. Brown and L. Shepp, 'A convolution inequality', preprint.

[5] G. Brown, 'Measures of algebraic sums of sets', in preparation.

[6] C. C. Graham and O. C. McGehee, Essays in commutative harmonic analysis (SpringerVerlag, Berlin, 1979).

[7] D. Hajela and P. Seymour, 'Counting points in hypercubes, isoperimetric inequalities and convolution measure algebras', preprint.

[8] R. R. Hall, 'A problem in combinatorial geometry', J. London Math. Soc. (2) 12 (1976), 315-319.

[9] G. H. Hardy, J. E. Littlewood, and G. Polya, Inequalilties (Cambridge University Press, London, 1951).

[10] D. S. Mitrinovic, Analytic inequalities (Springer-Verlag, Berlin, 1970).

[11] D. M. Oberlin, 'The size of sum sets, II', preprint. 
[12] M. Talagrand, 'Solution d'un probleme de R. Haydon', Publ. du Dept. de Math. Lyon 12-2 (1975), 43-46.

[13] D. R. Woodall, 'A theorem on cubes', Mathematika 24 (1977), 60-62.

School of Mathematics

University of New South Wales

Kensington, N.S.W. 2033

Australia 